\title{
Wireless Body Area Sensor Networks for Wearable Health Monitoring: Technology Trends and Future Research Opportunities
}

\author{
Malek ALRASHIDI ${ }^{1}$ \\ Department of Computer Science, Community College \\ University of Tabuk, Tabuk, KSA
}

\author{
Nejah NASRI ${ }^{2}$ \\ Laboratory of Electronics and Information Technology \\ (LETI), ENIS, Tunisia
}

\begin{abstract}
Today, there is an emerging interest in Wireless Body Area Sensor Networks (WBASNs) for the real-time monitoring of patients and early chronic disease detection. In this context, this paper presents a synopsis survey of healthcare monitoring via the IEEE 802.15.6 (UWB) protocol. We intend to propose a survey of the current issues of wearable physiological monitoring signals and devices, application areas, and reliability in WBASNs. To help elderly and disabled people, it would be beneficial to use a wireless transportable gadget at home to gather useful data in traditional human activities. This will manage regular hospital and emergency department appointments and will monitor crucial physiological signals realtime. This paper will also present a study on new wireless technologies intended for body area sensor networks, including signal processing problems, spectral allocation, security, and future research challenges of WBASNs.
\end{abstract}

Keywords-Healthcare; physiological signals; security; UWB; wireless technologies; WBASN

\section{INTRODUCTION}

Wireless Sensor Networks (WSNs) allow for better management of data collection in a healthcare context [1]. These include monitoring the patient's heart (electrocardiogram [ECG]), recording the electrical activity of the brain (electroencephalography [EEG]), evaluating the electrical activity produced by skeletal muscles (electromyogram [EMG]), and measuring the cornea-positive standing potential relative to the back of the eye (electrooculogram [EOG]). Physiological monitoring systems use an embedded WSN in the patient's biological tissue to regularly send the collected data to the base station, which performs the necessary processing to ensure the healthy functioning of vital organs [2]. These wireless technologies allow the person to permanently control these physiological parameters and their level of performance. These systems are used to send alarms to medical staff in the case of organ malfunction or abnormal parameter detection.

Existing signal control techniques used in hospitals cannot be used in transportable devices due to the following logic [3]:

- Regular systems for monitoring physiology are unwieldy and not healthy to wear for an extended period.
- There is loss of signal quality after extended use of the electrodes and gels.

- There are many cables used to transmit data to the sinks.

To resolve conflicts and difficulties in the field of controlling physiological signals, there is a need to develop wireless body area sensor network (WBASN) architecture, working on the invasive or non-invasive human body, to keep an eye on crucial health parameters.

A WBASN based on IEEE 802.15.6 standards [4] is a network composed of several vital signal sensing devices. The collected data from sensors are transmitted by wireless technologies to a medical server, then sophisticated applications analyze the data to make the right decisions based generally on artificial intelligence (machine learning, deep learning, and neuronal networks).

Technological advances in signal processing and the increasing level of integration for embedded systems have driven research into the engineering of new communication systems for the healthcare of elderly or disabled peoples, including WBASNs [5][6]. Figure 1 demonstrates the process of WBASN communication for health care monitoring.

Generally, communication in a WBASN goes through three steps [7]. The first step is the detection of vital organ parameters or physiological signals, such as blood oxygen level, blood pressure, ECG, EOG, and EEG. The second step is to transmit the collected data to the health server through a gateway using specific communication technologies, such as UWB, ZigBee, BLE, and Wi-Fi. The last step is responsible for classification, analysis, and early deduction of abnormalities. This step is achieved by using new artificial intelligence techniques, such as deep learning. Figure 2 shows a detailed description of the communication processes in WBASN.

In this paper, WBASN technologies are applied to critical healthcare monitoring research areas. In addition, the network architecture of WBASNs is presented. Resource allocation algorithms, routing, MAC protocols, security, and privacy are provided with qualitative comparisons. 


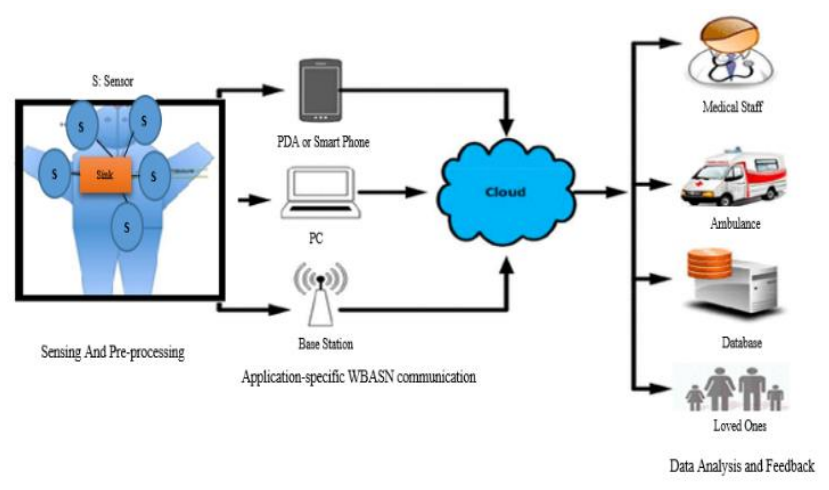

Fig. 1. Process of WBASN Communication.

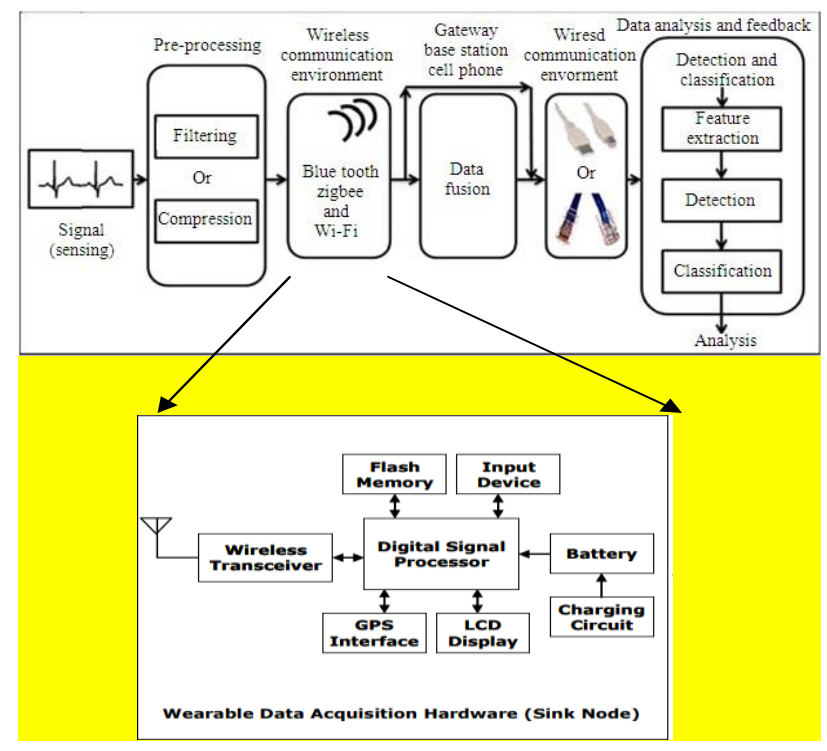

Fig. 2. Signal Processing and Communication in WBASN.

The rest of the paper is organized as follows. Section 2 introduces a synopsis of WBASN communication technologies. Section 3 explores WBASN architecture. Section 4 presents WBASN routing protocols. Finally, Section 5 challenges and explores issues in WBASNs.

\section{WBASN COMMUNICATION TECHNOLOGIES}

A WBASN is a sub-field of a WSN that interconnects sensor nodes or actuator capabilities in, on, or around a human body [8]. WPANs (wireless personal area network), like Bluetooth low energy (IEEE 802.15.1), Wi-Fi (based on 802.11b), UWB (Ultra-Wide Band) based on the 802.16.5 standard, and Zigbee (IEEE 802.1.5.4 Standard) can be used to ensure compatibility between nodes and the base station. All WBAN network architectures consist of several connected nodes, performing the tasks of communication detection and data processing.

\section{A. Bluetooth Low Energy (BLE 4.0-5.0)}

Bluetooth low energy is a specific technology characterized by low-power radio consumption in which applications using BLE can run on a small battery for many years. Despite traditional Bluetooth, BLE is designed to exchange data quickly and over long distances [9].
BLE operates in a $2.4 \mathrm{GHz}$ frequency band and can transmit data over 40 channels using CDMA spread spectrum techniques. Additionally, BLE supports data throughput from $125 \mathrm{~KB} / \mathrm{s}$ to $2 \mathrm{MB} / \mathrm{S}$, and the power consumption level does not exceed $100 \mathrm{mw}$.

Table I describes the two versions of BLE, 4.0 vs. 5.0.

TABLE I. COMPARISON OF BLUETOOTH V 4.0 vs. V 5.0

\begin{tabular}{|l|l|l|}
\hline & BLE 4.0 & BLE 5.0 \\
\hline Data rates & $125 \mathrm{~Kb} / \mathrm{s} \rightarrow 1 \mathrm{Mb} / \mathrm{s}$ & $125 \mathrm{~Kb} / \mathrm{s} \rightarrow 2 \mathrm{Mb} / \mathrm{s}$ \\
\hline Data ranges & 10 meters indoors & 40 meters indoors \\
\hline Support IoT devices & No & Yes \\
\hline Power consumption & High & Less \\
\hline Security control & Less (16-bit CRC) & High (24-bit CRC) \\
\hline Message length & $\approx 255$ bytes & $\approx 31$ bytes \\
\hline
\end{tabular}

B. ZigBee (IEEE 802.15.4)

ZigBee is a standard that needs a low-throughput, lowenergy consumption, cost-effective wireless tenders, an extended lifetime battery, and trusted networking [10]. Technically, ZigBee technology is simpler than other common wireless standards, such as Bluetooth, Wi-Fi, and UWB. Comparing ZigBee with other technologies and standards, UWB is the most suitable for WSNs because of its low power consumption. ZigBee is proposed for short-range communication and limited energy consumption. Consequently, it will not affect the battery lifetime.

ZigBee presents two frequency bands, $2.4 \mathrm{GHz}$ and 868/ $915 \mathrm{MHz}$. It gives $250 \mathrm{~KB} / \mathrm{s}$ throughput data for $2.4 \mathrm{GH} ; \mathrm{z}$ and 20 and $40 \mathrm{kbps}$ for 868 and $915 \mathrm{MHz}$, respectively.

\section{UWB (802.16.5)}

Ultra-wide band (UWB) technology is characterized by low power consumption, high data throughput, and short-range communication [11]. Due to the health monitoring applications and limited coverage area, the IEEE 802.15.6 standard defines the physical and MAC layers of UWB.

Table II describes the characteristics of the IEEE 802.15.6 standard.

A summary statement of Bluetooth (IEEE 802.15.1), ZigBee (IEEE 802.1.5.4), and UWB (802.16.5) technologies is presented [9-12] in Table III.

TABLE II. CHARACTERISTICS OF THE IEEE 802.15.6 STANDARD

\begin{tabular}{|l|l|}
\hline Data rate & Up to $10 \mathrm{Mb} / \mathrm{s}$ \\
\hline MAC techniques & $\begin{array}{l}\text { CSMA/CA } \\
\text { Slotted Aloha }\end{array}$ \\
\hline Communication range & $\approx 10$ meters \\
\hline \multirow{2}{*}{ Security } & $\begin{array}{l}\text { Three levels of security: communication level, } \\
\text { authentication level, encryption level }\end{array}$ \\
\hline & $402-405 \mathrm{MHz} ; 420-450 \mathrm{MHz} ;$ \\
Frequency Band & $863-870 \mathrm{Mhz} ; 902-928 \mathrm{MHz} ;$ \\
& $950-958 \mathrm{Mhz} ; 2360-2400 \mathrm{MHz} ;$ \\
& $2400-2485 \mathrm{MHz}$ \\
\hline
\end{tabular}


TABLE III. Technical Parameters of ZigBee, Bluetooth (BLE.5.0), AND UWB TECHNOLOGIES

\begin{tabular}{|c|c|c|c|}
\hline Standards & ZigBee & BLE 5.0 & UWB \\
\hline IEEE spec. & 802.15 .4 & 802.15 .5 & 802.15 .6 \\
\hline Frequency Band & $2.4 \mathrm{GHz}$ & $2.4 \mathrm{GHz}$ & $402 \mathrm{MHz} \rightarrow 2485 \mathrm{MHz}$ \\
\hline $\begin{array}{l}\text { Communication } \\
\text { range }\end{array}$ & $\begin{array}{l}\approx 30 \mathrm{~m} \\
\text { indoors }\end{array}$ & $\begin{array}{l}\approx 40 \mathrm{~m} \\
\text { indoors }\end{array}$ & $\approx 10 \mathrm{~m}$ indoors \\
\hline Number of channels & 27 & 40 & 27 \\
\hline CRC error detection & 16-bit & 24-bit & 32-bit \\
\hline Data rate & $250 \mathrm{~KB} / \mathrm{s}$ & $\begin{array}{l}\text { Up to } \\
2 \mathrm{MB} / \mathrm{s}\end{array}$ & $\begin{array}{l}\text { From } 10 \mathrm{~KB} / \mathrm{s} \text { to } 10 \\
\mathrm{Mb} / \mathrm{s} \text {. }\end{array}$ \\
\hline Topology & $\begin{array}{l}\text { star, tree, } \\
\text { mesh }\end{array}$ & $\mathrm{p} 2 \mathrm{p}$, star & p2p, star \\
\hline
\end{tabular}

The UWB approach is intended for applications with reduced distances that are high throughput, such as health monitoring. In contrast, Wi-Fi is designed for high range and sustains devices with a significant power supply. The emerging technology of wireless sensor networks (WSN) has become a new paradigm for factory automation systems and industrial monitoring. The control of data size has been generally kept small (e.g., the temperature data in environmental control requests fewer than four bytes). Bluetooth and ZigBee technologies have provided excellent results, despite their slow data rate.

\section{WBASN ARCHITECTURE}

A WBASN is a new generation of WSNs adapted to support and control the human body. A WBAN consists of a certain number of nodes related to the physiological signals being monitored. Each node of the network is equipped with a biomedical sensor, processing unit (ADC, processor, etc.), and communication unit that generally use UWB (IEEE 802.15.6 standard).

Each network node, whether invasive or non-invasive, collects vital signals from the human body (ECG, EEG, etc.) and transmits them to a base station (central unit that processes the data using artificial intelligence techniques (deep learning, machine learning, neural network).

In addition to sensing and sending data, the body nodes can be equipped with actuators, such as pacemakers, to stimulate the heartbeat or a drug injection device.

A WBASN is an example of a WSN with variable characteristics, such as type of node, density of nodes, data transfer rate, mobility, interoperability, and communication distance. Moreover, the number of nodes in a body network depends essentially on the number of signals and organs to be controlled. Generally, this number is limited. Spies [12] presented the architecture of heartbeat control.

It is typically stable regarding the data transmission rates since it has real-time control of the physiological signals. They are associated with the patients and move together in the exact directions with the same mobility speed.

The architecture of a WBAN follows two models: a traditional model, if it is a single patient to be controlled, and a distributed model, if it controls several patients in a hospital.
For each model, there are three types of communication: intra-, inter-, and beyond-WBAN [13].

For each type of communication, there is a specific architecture. For example, for intro-WBAN communication, the adequate architecture is centralized (star topology).

For inter-WBAN communication, the most frequently used architecture is mesh architecture to guarantee low energy consumption and a better QoS. Finally, for beyond-WBAN communication, a mesh architecture is maintained to guarantee a better QoS.

\section{A. Intra-WBAN Architecture}

On-body communication among biomedical sensor nodes is called intra-WBAN communication. As shown in Figure 3, the intra-WBAN structure is made up of an invasive cluster head $(\mathrm{CH})$ that collects data from invasive biomedical sensors in a star topology. $\mathrm{CH}$ relays information to the gateway and then to the medical server (MS) to process data. This architecture is done only for a single body [14].

\section{B. Inter-WBAN and Beyond-WBAN Architecture}

This architecture is designed especially for people or patients while exercising their quotidian routine, functioning indoors or outdoors.

Inter-WBAN architecture involves communication between two or more intra-WBANs [15] through a router or communication to transmit data to their destination, generally a medical server. In beyond-WBAN, architecture, authorized healthcare personnel (doctor, nursing orderly, etc.) have access to patients' medical information through the internet (cloud).

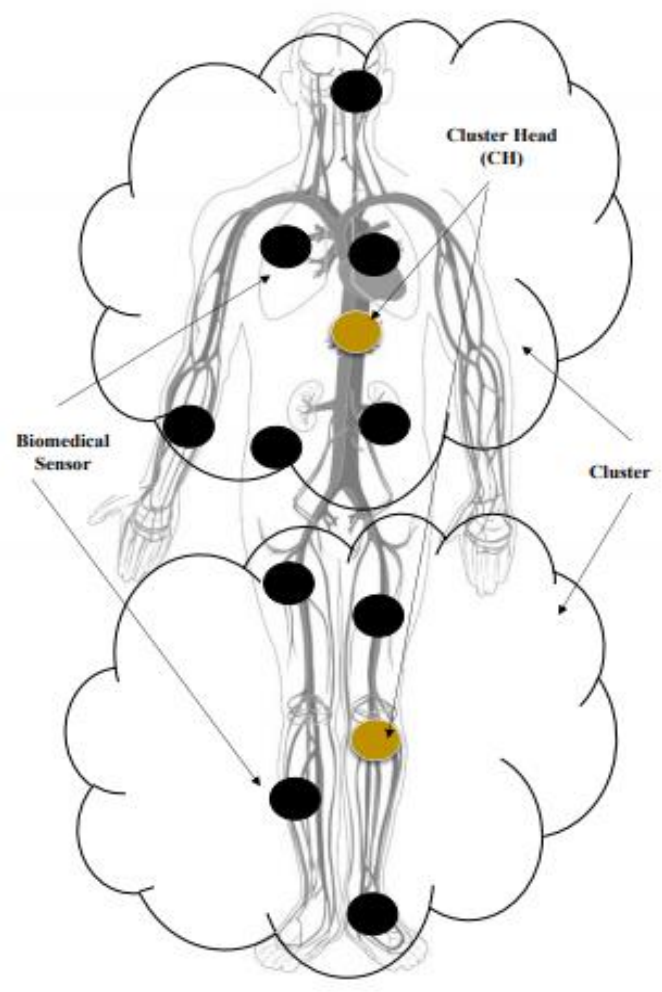

Fig. 3. Intra-WBAN Communication Architecture. 
Figure 4 describes Inter/beyond-WBAN communication.

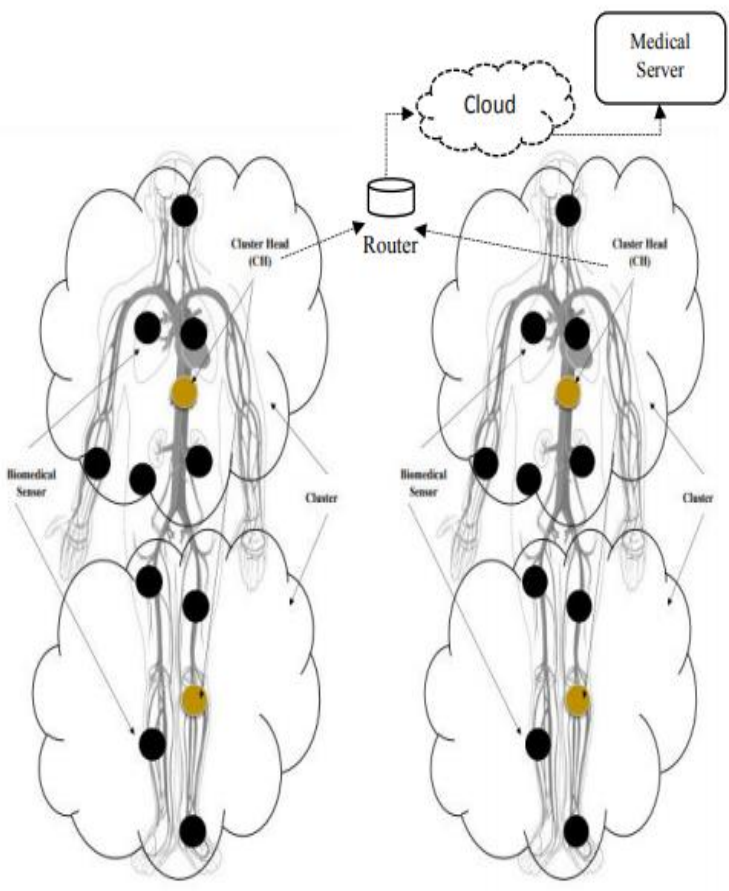

Fig. 4. Inter/Beyond-WBAN Communication Architecture.

\section{ROUTING IN WBASN}

The operating environment of a WBASN is very complex and specific, especially for the transmission of physiologically relevant data that directly impacts the human being's life and health [16]. For these and other reasons, several challenges and constraints must be considered when designing a routing protocol to ensure the reliability of WBASNs. Among these constraints, the communication channel's specificity, whether invasive for an intra-WBASN architecture or non-invasive for inter and/or beyond architecture, is cited. This makes it necessary to consider biological tissue parameters for the frequency choice, allowing low transmission loss and reducing interference.

In addition, a WBASN is implanted in the human body. Therefore, recharging and replacing the batteries requires surgical intervention, and, in many cases, it is not possible to replace these batteries. For these reasons, battery life must be considered when designing the routing protocol. Similarly, the body nodes' temperature must be considered to protect the human body's biological tissues and organs.

The quality-of-service requirements must also be considered, especially since the sensors' data are of different types, such as necessity data, delay-sensitive data, and general data. According to recent research, WBASN routing protocols can be classified according to energy-, cluster-, temperature-, cross-layer-, QoS-, and posture-based routing.

\section{A. Energy-based Routing}

Minimizing energy consumption is a fundamental objective to be met when building an efficient WBAN architecture [17].
Indeed, proposing a routing protocol that regards the residual energy at the battery level allows.

Similarly, a routing protocol must consider the SAR (specific absorption rate) to increase the network's lifetime to considerably save human biological tissue. Most routing protocols in the literature only consider transmission energy, which represents two-thirds of the energy consumed, and do not consider the effects of electromagnetic waves absorbed by the human body (EMRF), which results in a high SAR. High energy consumption primarily affects the human body. It increases SAR, which affects biological tissue, reduces blood flow (circulation), and affects enzymatic reactions.

Power consumption has consequences on the WBAN network architecture in terms of lifetime and reliability, as well as on communication in terms of interference (EMI), propagation delay, reliability, and attenuation.

\section{B. Cluster-based Routing}

Self-organizations are the most studied problem by researchers [18]. One of them is based on the creation of a backbone to optimize the diffusion of information. A second solution called distributed hash table (DHT) aims to recover dynamic information [19].

Another approach called clustering techniques is based on dividing the network into small areas, called clusters. Each area is managed by a sensor node, called a cluster head $(\mathrm{CH})$, with the aim being to optimize the network parameters. Several clustering algorithms have been defined. The election metric of $\mathrm{CHs}$ is not always the energy parameter, but node degree and mobility are also considered.

Much research has shown that the clustering techniques used for WSNs are well suited for WBASNs. Furthermore, many clustering techniques try to minimize direct communication between a biomedical node and the server to minimize the communication distance, subsequently reducing the communication energy dissipated by the transmitter.

\section{Temperature-based Routing}

An invasive biomedical node heats up during operation, which can damage vital human tissues and organs. Therefore, the temperature must be considered during the design of the routing protocols to avoid these problems through a node with a high temperature (Figure 5).

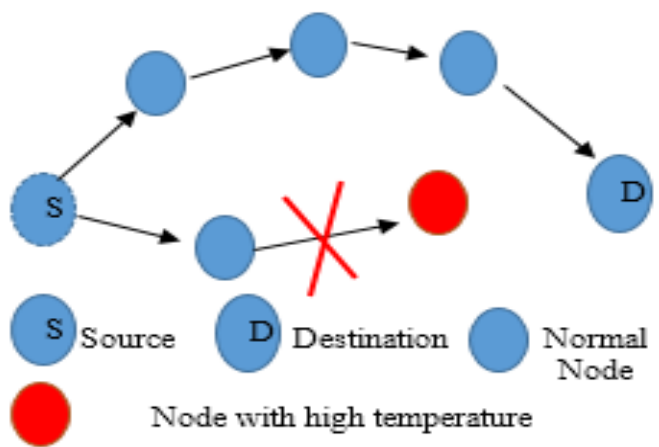

Fig. 5. Temperature-based Routing [20]. 


\section{Cross Layer-based Routing}

Cross-layer protocol refers to crossing or merging the functionalities of two or more layers in the protocol pile. The design of a routing protocol based on the cross-layer improves energy efficiency, interoperability between layers, QoS, and flow, according to the communication channel's characteristics. Typically, there are some protocols, such as thermal-aware routing algorithms and adaptive least temperature routing, named M-ATTEMPT [21].

The protocols are classified according to the layers of the protocol pile involved in the cross-layer strategy (physical and MAC layers; physical and network layers; physical, MAC, and network layers; physical link and network; physical link and application; physical network and application).

1) Physical and MAC layers: The objective of the interaction between these two layers is synchronization between the access techniques to the communication medium and the characteristics of the transceivers in terms of transmission power, SNR, and the energy level available to the batteries. For example, in the work presented in [22], energy-efficient cross-layer optimization was used for wireless personal area networks (WPAN), which were then deployed to WBANs, where the SNR value depended on the ARQ (automatic repeat request) of the MAC layer to increase energy efficiency.

2) Physical and network layers: SNR value, battery level, and electronic characteristics of the transceiver are considered when designing a routing protocol. It is an interaction between the physical layer and the network layer.

In [23], proposed a priority-based cross-layer routing protocol for healthcare uses. This protocol results from the interaction between the MAC layer and the network layer for invasive and non-invasive body communication. This work showed the reliability and stability of network reliability, throughput, QoS, and energy performance. In [25][26], protocols were proposed for these two layers to interact, improving network density and QoS.

\section{E. Posture-based Routing}

WBAN nodes are often disconnected from the network due to posture movement. Therefore, researchers regularly update a cost function that chooses the best path of packets to the sink. An example of a posture-based routing protocol is the on-body store and flood protocol, which guarantees better routing of information and low energy consumption based on multi-hop routing [24].

\section{F. QoS-based Routing}

The growing demand for real-time WBASN applications to support the elderly, disabled, and other patients is exponentially driving the need for QoS-based protocols. Moreover, the design of QoS-based routing protocols has a significant influence on the efficiency of WBASNs [25].

In a WBASN network, physiological signals have a direct impact on the health of the human being. For this reason, the QoS must consider the type of data to be transmitted.
Depending on the application, the routing process can integrate several QoS measures, such as propagation delay, energy consumption, and reliability.

In [26], a taxonomy of QoS-based routing protocols in WBASNs was presented. In addition, this work summarized the advantages and disadvantages of each routing protocol and presented a benchmark survey of all QoS-based routing protocols found in the literature.

\section{OPEN ISSUES AND FUTURE RESEARCH}

The increasing number of WBASN users and the growing number of wireless biomedical devices reveal several research topics and challenges for improving WBASNs. In this work, some open issues and future research directions, as described in [27][28][29], are presented.

\section{A. Channel Allocation}

The WBASN network's commercialization has increased the number of users, especially since the application areas are very diverse.

The network traffic in the communication channel increases with the number of users, which has augmented the collision rate, the loss of information, and the interference between the sub-channels for the different technologies used, such as UWB, ZigBee, and Bluetooth. The communication channel designed for WBAN may not meet future requirements.

A study by the Federal Communications Commission (FCC) has shown that specific frequency bands are partially occupied. Thus, cognitive radio (CR) can be a solution for the optimal utilization of communication channels.

$\mathrm{RC}$ is an intelligent communication technique that identifies and detects the used channel and moves communication to unused channels. CR optimizes the use of the signal spectrum to reduce interference between users.

The ISM band has become unable to support the requirements of WBASN, hence the idea of using unlicensed frequency bands outside the ISM band.

\section{B. Network Security}

A WBASN is a new technological trend allowing real-time control and monitoring of patients' health status. The security and protection of data against intrusions from detection to medical server routing is a crucial challenge for researchers in the field.

A WBASN architecture must use security measures that guarantee the confidentiality of medical records.

The main security and privacy challenges for a WBASN infrastructure are data authentication, confidentiality, integrity, freshness, availability of the network, secure management and localization, dependability, accountability, flexibility, privacy rules, and compliance requirements.

Security and confidentiality are the main challenges in WBASN architecture. Currently, several current research works focus on this problem, including privacy and quality of service (QoS), trust management, and integrating WBANs with mobile phones. 


\section{Radiofrequency Safety}

For the internet of things, $5 \mathrm{G}$ refers to developments in terms of speed and services. The uses of $5 \mathrm{G}$ are diverse and varied, for example, the enrichment of the connected home, autonomous vehicle, and arrival of Health 4.0 (WBASN).

The commercialization of $5 \mathrm{G}$ has increased the number of electronic devices used in a body network. This has increased the rate of radiofrequency radiation (RF), causing electromagnetic interference (EMI), affecting sensor devices, and risking patient safety.

Despite standardization, the exposure limits to RF radiation must be reviewed because of the exponential increase of radiating devices with the commercialization of $5 \mathrm{G}$ and IoT service integration.

\section{WBASN Service Standardization}

WBAN technology commercialization requires interoperability between the different suppliers' electronic devices in the OSI model's layers.

Network scalability of WBAN devices should consider the maximum number of body sensors, energy consumption, packet losses, throughput, and synergic integration with 5G mobile communication.

\section{E. Energy Consumption}

WBASNs are used in the primary interest of human health (patient monitoring, disease detection, elderly monitoring, etc.). For this reason, reliability is a significant challenge. In contrast, energy consumption must be considered to guarantee the reliability of the network.

This parameter introduces the problem of energy conservation, especially when the application operates for a long time. Usually, it is not common for body sensor networks to recharge or replace node batteries after being depleted.

To increase the lifetime of a network or optimize the transmission parameters, research has proposed designing a virtual structure based on the phenomenon of self-organization. The main goal of self-organization is to minimize the transfer disorder in the network and improve the sensors' performance.

\section{CONCLUSION}

In this study, a summary of the new collection of WSNs called WBASN is presented. There is a need for this technology for patient monitoring and maintenance in elderly and disabled people. Architecture routing protocols and a comparative survey between technology related to WBASN (ZigBee, BLE, UWB) are cited.

Additionally, problems related to the WBASN network and research directions to strengthen this technological trend for service integration with $5 \mathrm{G}$ mobile communication and a synergic integration with Industry 4.0 are presented.

\section{REFERENCES}

[1] WU, Changcheng, et al. A low cost surface EMG sensor network for hand motion recognition. In: 2018 IEEE 1st International Conference on Micro/Nano Sensors for AI, Healthcare, and Robotics (NSENS). IEEE, 2018. p. 35-39.
[2] OMODUNBI, Bolaji A., et al. Wireless sensor network based health monitoring system for hypertensive in-patients. FUOYE J. Eng. Technol, 2018, 3.2: 6.

[3] DARWISH, Ashraf; HASSANIEN, Aboul Ella. Wearable and implantable wireless sensor network solutions for healthcare monitoring. Sensors, 2011, 11.6: 5561-5595.

[4] MARTELLI, Flavia; BURATTI, Chiara; VERDONE, Roberto. On the performance of an IEEE 802.15. 6 wireless body area network. In: 17th European Wireless 2011-Sustainable Wireless Technologies. VDE, 2011. p. 1-6.

[5] NASRI, Nejah, et al. Efficient encoding and decoding schemes for wireless underwater communication systems. In: 2010 7th International Multi-Conference on Systems, Signals and Devices. IEEE, 2010. p. 1-6.

[6] NICOLAU, Hugo; MONTAGUE, Kyle. Assistive technologies. In: Web Accessibility. Springer, London, 2019. p. 317-335.

[7] NEGRA, Rim; JEMILI, Imen; BELGHITH, Abdelfettah. Wireless body area networks: Applications and technologies. Procedia Computer Science, 2016, 83: 1274-1281.

[8] HÄMÄLÄINEN, Matti; LI, Xinrong. Recent advances in body area network technology and applications. International Journal of Wireless Information Networks, 2017, 24.2: 63-64.

[9] FOURATI, Lamia Chaari; SAID, Sana. Remote Health Monitoring Systems Based on Bluetooth Low Energy (BLE) Communication Systems. In: International Conference on Smart Homes and Health Telematics. Springer, Cham, 2020. p. 41-54.

[10] S. Mnasri, N. Nasri and T. Val, "An Overview of the deployment paradigms in the Wireless Sensor", Networks International Conference on Performance Evaluation and Modeling in Wired and Wireless Networks (PEMWN 2014), November 04-07th, 2014.

[11] DE SANTIS, Valerio; FELIZIANI, Mauro; MARADEI, Francescaromana. Safety Assessment of UWB Radio Systems for Body Area Network by the $\$\{\backslash \mathrm{rm} \text { FD }\}^{\wedge}\{2\}\{\backslash \mathrm{rm}$ TD $\}$ M Method. IEEE Transactions on Magnetics, 2010, 46.8: 3245-3248.

[12] SPIES, Chel-Mari. Proposed model for evaluation of mHealth systems. In: 2015 International Conference on Computing, Communication and Security (ICCCS). IEEE, 2015. p. 1-8.

[13] JABEEN, Tallat; ASHRAF, Humaira; ULLAH, Ata. A survey on healthcare data security in wireless body area networks. Journal of Ambient Intelligence and Humanized Computing, 2021, 1-14.

[14] AL-JANABI, Samaher, et al. Survey of main challenges (security and privacy) in wireless body area networks for healthcare applications. Egyptian Informatics Journal, 2017, 18.2: 113-122.

[15] ALI, Aftab; KHAN, Farrukh Aslam. Energy-efficient cluster-based security mechanism for intra-WBAN and inter-WBAN communications for healthcare applications. EURASIP Journal on Wireless Communications and Networking, 2013, 2013.1: 1-19.

[16] UL HUQUE, Md Tanvir Ishtaique, et al. EAR-BAN: energy efficient adaptive routing in wireless body area networks. In: 2013, 7th International Conference on Signal Processing and Communication Systems (ICSPCS). IEEE, 2013. p. 1-10.

[17] SAGAR, Anil Kumar; SINGH, Shivangi; KUMAR, Avadhesh. Energyaware WBAN for health monitoring using critical data routing (CDR). Wireless Personal Communications, 2020, 1-30.

[18] MU, Jiasong, et al. A self-organized dynamic clustering method and its multiple access mechanism for multiple WBANs. IEEE Internet of Things Journal, 2018, 6.4: 6042-6051.

[19] PUNJ, Roopali; KUMAR, Rakesh. CHS-GA: An approach for cluster head selection using genetic algorithm for WBANs. In: Online Engineering \& Internet of Things. Springer, Cham, 2018. p. 28-35.

[20] AHMED, Ghufran; MAHMOOD, Danish; ISLAM, Saiful. Thermal and energy aware routing in wireless body area networks. International Journal of Distributed Sensor Networks, 2019, 15.6: 1550147719854974.

[21] AHMAD, Ashfaq, et al. RE-ATTEMPT: a new energy-efficient routing protocol for wireless body area sensor networks. International Journal of Distributed Sensor Networks, 2014, 10.4: 464010.

[22] CORREA-CHICA, Juan Camilo; BOTERO-VEGA, Juan Felipe; GAVIRIA-GÓMEZ, Natalia. Cross-layer designs for energy efficient 
wireless body area networks: a review. Revista Facultad de Ingeniería Universidad de Antioquia, 2016, 79: 98-117.

[23] ELHADJ, Hadda Ben, et al. A priority based cross layer routing protocol for healthcare applications. Ad Hoc Networks, 2016, 42: 1-18.

[24] NABI, Majid; GEILEN, Marc; BASTEN, Twan. MoBAN: A configurable mobility model for wireless body area networks. In: Proceedings of the 4th international ICST conference on simulation tools and techniques. 2011. p. 168-177.

[25] KHAN, Zahoor A., et al. A QoS-aware routing protocol for reliability sensitive data in hospital body area networks. Procedia Computer Science, 2013, 19: 171-179.

[26] QU, Yating, et al. A survey of routing protocols in WBAN for healthcare applications. Sensors, 2019, 19.7: 1638.
[27] LIU, Qingling; MKONGWA, Kefa G.; ZHANG, Chaozhu. Performance issues in wireless body area networks for the healthcare application: a survey and future prospects. SN Applied Sciences, 2021, 3.2: 1-19.

[28] CICIOĞLU, Murtaza; ÇALHAN, Ali. Channel aware wireless body area network with cognitive radio technology in disaster cases. International Journal of Communication Systems, 2020, 33.16: e4565.

[29] OLATINWO, Damilola D.; ABU-MAHFOUZ, Adnan M.; HANCKE, Gerhard P. Towards achieving efficient MAC protocols for WBANenabled IoT technology: a review. EURASIP Journal on Wireless Communications and Networking, 2021, 2021.1: 1-47. 